\section{Parakristallines Endoplasmatisches Retikulum im Ovar von Pyrrhocoris apterus (Heteroptera)}

\author{
U. MAYS *
}

Zoologisches Institut der Universität Münster (Westf.)

(Z. Naturforschg. 22 b, 459 [1967] ; eingegangen am 2. Januar 1967)

In den telotroph-meroistischen Ovarien der Feuerwanze Pyrrhocoris apterus L. kommen Komplexe des Endoplasmatischen Retikulums (ER) in parakristalliner Ordnung vor. Sehr ähnliche Zustände des ER wurden von Eymé ${ }^{1-3}$ in Samenanlagen und Nektarien gefunden und wegen des Aspekts der Schnittbilder als „cotte de maille“ (Panzerhemd) bezeichnet. Gleichartige Gebilde hat auch BAssoT ${ }^{4,5}$ in den Leuchtzellen mariner Anneliden eingehend untersucht.

Das Parakristalline Endoplasmatische Retikulum (PkER) von Pyrrhocoris bildet Komplexe bis zu $5 \mu \mathrm{m}$ Größe. Diese finden sich regelmäßig unterhalb der Einmündung des Nährstrangs in Oozyten, die sich noch im euplasmatischen Wachstum befinden, also vor Beginn der Dottereinlagerung stehen (Abb. 1)*. Weiterhin kommt PkrER in dem Plasmahof vor, der zwischen den Nährzellen des telotrophen Ovars im Germarium liegt. Dort bildet es z. T. größere zusammengesetzte Komplexe nach Art eines Vielkristalls. Im Nährstrang tritt PkER nur vereinzelt auf. Im Bereich der parakristallinen Komplexe des ER finden sich zwei durch eine Membran voneinander getrennte Räume; der erste mit etwas weiterem Lumen ist der Binnenraum (Zisterne) des ER, und ein zweiter, englumiger, hat Anschluß an das Cytoplasma (Abb. $2 \rightarrow$ ). Beide durchdringen einander derart, daß jeder für sich ein kontinuierliches räumliches Netzwerk bildet und jeder die Lücken im Netz des anderen ausfüllt. Das Ganze bildet ein regelmäßiges Raumgitter mit einem für das Cytoplasma erstaunlich hohen Ordnungsgrad. Die Identitätsperiode auf Schnitten parallel zu einer Kristallachse beträgt etwa $1550 \AA$, das Lumen des engen Raumes 210 bis $270 \AA$. Bei Schnitten, die nicht parallel zu den Kristallachsen verlaufen, erscheint die Identitätsperiode bis auf $2000 \AA$, bzw. das Lumen des engen Raumes stellenweise bis auf $300 \AA$ verlängert. Es konnte noch kein

* Die Untersuchungen laufen weiter; sie werden aus Mitteln unterstützt, die die Deutsche Forschungsgemeinschaft Herrn Prof. Bier zur Verfügung stellte. Herrn Doz. Dr. F. AmeLUnXen danken wir sehr für Arbeitsmöglichkeit am Elektronenmikroskop.

1 J. EyмÉ, Botaniste 45, 163 [1962].

2 J. Eymé, C. R. hebd. Séances Acad. Sci. 262, 1629 [1966]. voll befriedigendes Modell der beiden einander durchdringenden Raumgitter konstruiert werden.

Der von Bassot für das PkER der Anneliden gemachte Strukturvorschlag geht von Serien von Mikrotubuli aus, die etwa eine Sinuskurve nachzeichnen. Die Periode beträgt $800 \AA$ und der Durchmesser der Mikrotubuli $200 \AA$ A . Die Tubuli einer Ebene verlaufen parallel und haben gleiche Phase. Die der nächstfolgenden Ebene sind dagegen um eine halbe Phase und eine halbe Amplitude verschoben. Ring- und achtförmige Bilder werden als Überlagerung der Mikrotubuli zweier aufeinanderfolgender Ebenen in einem Schnitt erklärt. Nur an den Grenzen des Komplexes findet Bassot Verbindungen der Mikrotubuli verschiedener Ebenen und rechnet mit der Möglichkeit, daß es sich innerhalb des Parakristalls um nur wenige hin und herlaufende Tubuli handelt. Dieser Modellvorschlag scheidet für Pyrrhocoris aus, da die Vernetzung der Membran unter bestimmten Schnittwinkeln des PkER ganz deutlich wird. Es müssen die vielen Membranpartien berücksichtigt werden, die in die Schnittebene einbiegen und die um so unschärfer begrenzt erscheinen, je stärker sich der Verlauf der Membranebene dem der Schnittebene nähert (Abb. $2>, 3$ ). Eine Eindruck von dem hohen Vernetzungsgrad der englumigen Komponente des Raumgitters liefert Abb. 3, während in Abb. 2 der Zusammenhang im weitlumigen Anteil entlang einer Kristallachse unmittelbar ersichtlich ist.

Das PkER von Pyrrhocoris ist an der Einmündungsstelle des Nährstrangs in die Oozyte konzentriert. Die große Zahl der ER-Schläuche, die den Nährstrang exakt parallel zu seiner Richtung vom Plasmahof bis zur Oozyte durchziehen, legt nahe, im PkER einen Speicher für ER-Inhalt und/oder -Membranen zu vermuten, da beide aus dem Bereich der Nährzellen fortwährend an die Oozyten abgegeben werden. Für eine Stoffwechselfunktion spricht andererseits die große innere Oberfläche (vgl. l.c. ${ }^{5}$ ), welche die beiden Kompartimente mehr verbindet als trennt. Die Tatsache, daß PkER in sehr verschiedenen Zellen und an so weit voneinander entfernten Stellen im Organismenbereich auftritt, macht weitere Untersuchungen erforderlich, ehe Präzises über die Funktion gesagt werden kann.

3 J. Exmé et M. Le Blanc, C. R. hebd. Séances Acad. Sci. 256, 4958 [1963].

4 J.-M. Bassot, C. R. hebd. Séances Acad. Sci. 259, 1549 [1964].

3 J.-M. BAssot, J. cellular Biol. 31, 135 [1966].

* Abbn. 1-3 s. Tafel S. 458 a.

Abb. 3. Parakristallines Endoplasmatisches Retikulum, besonders deutliche Vernetzung durch sehr viele flach getroffene Membranen. Technik wie Abb. 1. Vergrößerung: 43300:1.
Abb. 1. Mündung des Nährstrangs (NS) in die Oozyte, darunter Komplexe des Parakristallinen Endoplasmatischen Retikulums. $\mathrm{FE}=$ Follikelepithel. Technik: $\mathrm{OsO}_{4}$-Fixierung, Eponeinbettung über Propylenoxid. Vergrößerung: 75 : 1.

Abb. 2. Parakristallines Endoplasmatisches Retikulum, fast parallel zu einer Kristallachse geschnitten. Anschluß des englumigen Raumes an das Cytoplasma $\rightarrow$ und Vernetzung durch in der Schnittebene liegende Membranen $>$. Technik wie Abb. 1. Vergrößerung: 41800:1. 